\title{
Gain of function in Mycobacterium bovis BCG Moreau due to loss of a transcriptional repressor
}

\author{
Renata Monteiro-Maia', Paloma Rezende Correa', Periela da Silva Sousa-Vasconcelos², \\ Rosa Teixeira de Pinho², Leila Mendonça-Lima ${ }^{1 /+}$ \\ ${ }^{1}$ Fundação Oswaldo Cruz-Fiocruz, Instituto Oswaldo Cruz, Laboratório de Genômica Funcional e Bioinformática, Rio de Janeiro, RJ, Brasil \\ ${ }^{2}$ Fundação Oswaldo Cruz-Fiocruz, Instituto Oswaldo Cruz, Laboratório de Imunologia Clínica, Rio de Janeiro, RJ, Brasil
}

The Bacille Calmette-Guérin (BCG) vaccine comprises a family of genetically different strains derived by the loss of genomic regions (RDs) and other mutations. In BCG Moreau, loss of RD16 inactivates $r v 3405 c^{*}$, encoding a transcriptional repressor that negatively regulates the expression of Rv3406, an alkyl sulfatase. To evaluate the impact of this loss on the BCG and host cell viability and the cytokine profile, THP-1 cells were infected with BCG Moreau (harbouring the empty vector) and a complemented strain carrying a functional copy of $r v 3405 c$. Viability of the host cells and bacteria as well as the pattern of cytokine secretion were evaluated. Our results show that the viability of BCG Moreau is higher than that of the complemented strain in an axenic medium, suggesting a possible functional gain associated with the constitutive expression of Rv3406. Viability of the host cells did not vary significantly between recombinant strains, but differences in the profiles of the cytokine secretion (IL-1 $\beta$ and IL-6) were observed. Our results suggest an example of a functional gain due to gene loss contributing to the elucidation of the impact of RD16 on the physiology of BCG Moreau.

Key words: BCG Moreau - rv3405C - RV3406 - THP-1 cells - tuberculosis

Tuberculosis (TB) is caused by the intracellular pathogen Mycobacterium tuberculosis (Mtb) and remains a major public health problem. According to World Health Organization (WHO), 6.3 million new cases of TB were reported in 2016, ${ }^{(1)}$ and in the last two decades, the disease was responsible for more deaths than smallpox, malaria, plague, influenza and AIDS together. (2) Brazil remains among the high burden countries with an incidence of 42/100,000 inhabitants in 2016.(3)

Bacille Calmette-Guérin (BCG) is the only available vaccine against $\mathrm{TB}$ and the most widely used vaccine worldwide. ${ }^{(4)}$ Modern BCG comprises a group of different vaccine strains; $;^{(5,6)}$ all strains are derived from an original attenuated "BCG" obtained after sequential in vitro passages of a virulent Mycobacterium bovis isolate in a medium containing ox bile. (7) The attenuated strain was successfully used in humans for the first time in 1921 and, after 1925, was distributed to various laboratories worldwide and maintained under different culture conditions. ${ }^{(8,9)}$. By the time standardised seed-lot production methods were implemented in the 1960s, BCG sub-strains had accumulated a variety of specific genetic differences that partially explain the variable efficacy of the vaccine. ${ }^{(10)}$

BCG Moreau, the Brazilian vaccine strain, ${ }^{(11)}$ has a specific genomic deletion (RD16) comprising 7,608 bp ${ }^{(6)}$

doi: 10.1590/0074-02760180267

Financial support: CAPES, CNPq, FIOCRUZ

*The Mycobacterium tuberculosis H37Rv gene and protein nomenclature

will be used throughout the text for simplification.

+ Corresponding author: Imlima@ioc.fiocruz.br

Received 31 May 2018

Accepted 11 September 2018 resulting in the fusion of $r v 3400-r v 3405 c$ and loss of the intervening genes. ${ }^{(12)}$ Previous studies by our group have established that Rv3405c is a transcriptional regulator that represses the expression of the adjacent $r v 3406$ gene. ${ }^{(13)}$ As a consequence, BCG Moreau accumulates Rv3406 due to its constitutive expression; in comparison, BCG Pasteur does not produce Rv3406 under the standard culture conditions. Complementation of BCG Moreau with a functional copy of $r v 3405 c$ obtained from BCG Pasteur restores the regulation and abolishes the Rv3406 accumulation. ${ }^{(13)}$

The rv3406 sequence encodes a protein identified as an alkyl sulfatase. ${ }^{(14)}$ Sulfatases catalyse the hydrolysis of various molecules ranging from small cytosolic steroids (oestrogen sulfate) to complex cell-surface carbohydrates (glycosaminoglycans - GAG). These substrates contain a wide variety of sulfate esters including hydrophobic glucosinolate, steroid and thyronine sulfates, amphiphilic sulfated carbohydrates found in GAGs, proteoglycans, glycolipids and water-soluble mono- and disaccharide sulfates. ${ }^{(15)}$

The aim of the present study is to evaluate the functional impact of the loss of Rv3405c on the M. bovis BCG Moreau viability and its initial interaction with the host cells using a THP-1 cell model, monitoring the behaviour of the bacilli and the host cells in response to infection. Two BCG Moreau recombinant strains ${ }^{(13)}$ were compared in this study: the first strain $(\mathrm{M}:: 05 c)$ harbours a functional copy of $r v 3405 c$ from the BCG Pasteur cloned in the pUS972 plasmid, ${ }^{(16)}$ and the second strain carries the empty vector (MD05c). Both strains were grown in the $7 \mathrm{H} 9$ medium supplemented with $10 \%$ ADC and $0.05 \%$ Tween- 80 in the presence of $25 \mu \mathrm{g} \mathrm{mL}^{-1}$ kanamycin. For growth curves, three aliquots of each strain were defrosted and maintained independently for two weeks of culture expansion. Bacteria concentration 
was adjusted to an optical density $\left(\mathrm{OD}_{600 \mathrm{~mm}}\right)$ of 0.2 , and culture volumes were expanded to $60 \mathrm{~mL}$ of $7 \mathrm{H} 9 \mathrm{me}-$ dium. OD was evaluated every $24 \mathrm{~h}$ for two weeks.

Cells of a human myelomonocytic cell line, THP-1, were grown as suspension cultures in RPMI medium supplemented with $10 \%$ heat-inactivated foetal bovine serum (FBS) (Gibco), $100 \mu \mathrm{g} \mathrm{mL}^{-1}$ penicillin and $100 \mu \mathrm{g}$ $\mathrm{mL}^{-1}$ streptomycin (Sigma), $25 \mathrm{mM}$ Hepes (Sigma) and $2 \mathrm{mmMol} \mathrm{L}{ }^{-1}$ glutamine (Sigma) at $37^{\circ} \mathrm{C}$ in a humidified atmosphere of $5 \% \mathrm{CO}_{2}$. THP-1 cells were differentiated into macrophages using $200 \mathrm{nM}$ of phorbol 12-myristate 13 -acetate (PMA; Sigma) for $48 \mathrm{~h}$ followed by a $48 \mathrm{~h}$ incubation in the medium without PMA (protocol adapted from Daigneault et al..$\left.^{(17)}\right)$. Cells were infected at a multiplicity of infection (moi) of $1 / 1$ with each BCG strain for $4 \mathrm{~h}$ and then washed thrice with RPMI to remove noninternalised bacteria. No differences in internalisation rates were observed between the recombinant strains [Supplementary data (Fig. 1)]. At specific time points, samples were collected for bacterial and cell viability determination and cytokine assays.

Bacterial intracellular viability was evaluated at 4, 6 , $24,48,72$ and $96 \mathrm{~h}$ after the infection of $2 \times 10^{5}$ THP-1 cells. At these time points, cells were lysed with $0.05 \%$ SDS and bacteria were recovered after centrifugation for $10 \mathrm{~min}$ at $16.000 \mathrm{xg}$. For CFU determination, bacteria in the pellet fraction were serially diluted and plated on the 7H10 medium supplemented with $10 \%$ ADC and $25 \mu \mathrm{g}$ $\mathrm{mL}^{-1}$ kanamycin; the colonies were counted after 28-30 days of incubation at $37^{\circ} \mathrm{C}$.

To evaluate macrophage viability during the course of infection, $3.2 \times 10^{4}$ cells were plated onto black 96-well culture plates. At 24, 48, 72 and $96 \mathrm{~h}$ after the infection, $100 \mu \mathrm{L}$ of PrestoBlue diluted 1:10 (Life Technologies) was added to the infected cells for $1 \mathrm{~h}$, and then viability was evaluated with a FlexStation 3 microplate reader. Cells incubated with $0.01 \%$ Triton X-100 were used as zero viability controls; non-infected cells were used as a positive control and medium was used as a negative control.

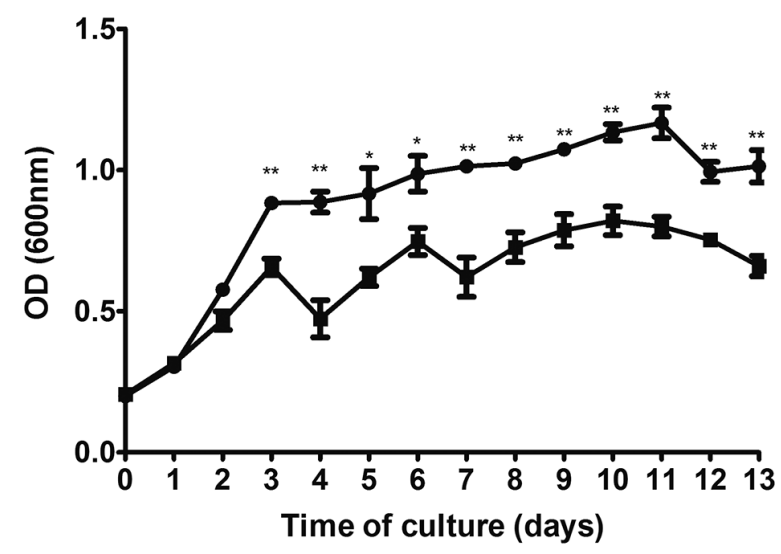

Fig. 1: comparison of growth rate of recombinant Bacille CalmetteGuérin (BCG) strains MD05c and M::05c in axenic medium for two weeks. Circles $(\bullet)$ indicate the MD05c strain, and squares ( $\bullet$ ) indicate the M::05c strain. Data represent the average of three biological replicates $+/$ - SD. T-Test $=* \mathrm{p}<0.05 ; * * \mathrm{p}<0.01$.
To evaluate cytokine secretion, $3.2 \times 10^{4}$ cells were plated onto 96-well culture plates and infected with each recombinant BCG strain as described above; at specific time points $(6,24$ and $72 \mathrm{~h})$, supernatants were removed, centrifuged for $2 \mathrm{~min}$ at $700 \mathrm{x} \mathrm{g}$ at $4^{\circ} \mathrm{C}$ and stored at $-70^{\circ} \mathrm{C}$ for subsequent cytokine and chemokine analyses. The production of the cytokines (IL-1 $\beta$, IL-6, IL-10 and TNF- $\alpha$ ) and of the chemokine MIP-1 $\beta$ was measured by enzyme-linked immunosorbent assay (ELISA) using a duo-set kit from R\&D Systems (cat nos: IL-1 $\beta$, DY 201; IL-6, DY 206; IL-10, DY 217B; TNF- $\alpha$, DY210; MIP-1 $\beta$, DY 271) according to the manufacturer recommendations. The limits of the detection of the cytokines were as indicated by the manufacturer: IL- $1 \mathrm{~b}=3.91-250 \mathrm{pg}$ $\mathrm{mL}^{-1}$; IL-6 $=9.38-600 \mathrm{pg} \mathrm{mL}^{-1}$; IL-10 $=31.2-2000 \mathrm{pg}$ $\mathrm{mL}^{-1}$; TNF- $\alpha=15.6-1000 \mathrm{pg} \mathrm{mL}^{-1}$; and MIP-1b $=15.6-$ $1000 \mathrm{pg} \mathrm{mL}^{-1}$. Spectrophotometer (Spectra Max 190) readings were taken at $450 \mathrm{~nm}$.

Statistical differences were calculated by T-test or two-way ANOVA and Bonferroni post-test (as specified in figure legends) using the Graph Pad Prism 5.0 Software (San Diego, CA, USA). Graphs represent at least three independent experiments. Data were considered significant if $\mathrm{p}<0.05$.

Comparison of the growth profiles of two BCG strains in an axenic medium shows a significant difference after three days and up to 13 days of culture, indicating a growth advantage for the strain $\mathrm{MD} 05 c$ that lacks the functional repressor (Fig. 1).

Ability to survive and multiply in an intracellular environment was assessed by infecting the THP-1 cells. At specific time points after the infection, the macrophages were lysed, bacteria recovered and CFUs determined by plating of serial dilutions as described above. The kinetics of viability indicates that, although MD $05 c$ lacks the functional repressor, it shows higher viability than M::05c; no statistically significant differences between the strains were observed (Fig. 2). Nevertheless, the data suggest that the presence of the functional Rv3405c tran-

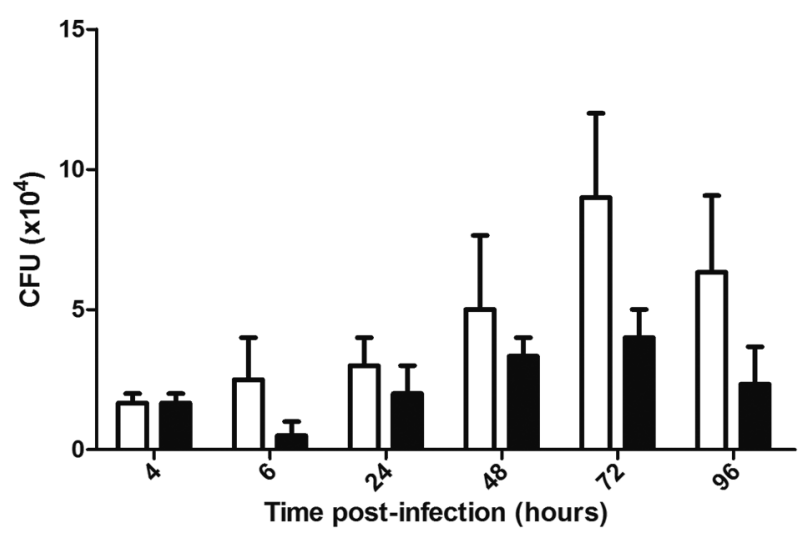

Fig. 2: intracellular viability of recombinant Bacille Calmette-Guérin strains (BCGs) in the THP-1 cells. Macrophages were infected with recombinant BCGs for 4 hours, and bacterial viability was evaluated at specific time points post-infection. No significant differences were observed. White bars represent the MD05c strain, and black bars represent the M::05c strain. The results represent the average of three independent experiments +/- SD. Non-paired T-test. 
scriptional repressor impacts intracellular survival of BCG; however, this finding should be confirmed in additional experiments to become statistically significant.

Our results show a significant impact on macrophage viability due to the infection with BCG when compared to the non-infected cells, but no significant differences could be associated with specific BCG strain used (Fig. 3).

Cytokine responses were evaluated in the host cells infected with two recombinant BCGs and non-infected THP-1 cells. The levels of secreted IL-10, MIP-1 $\beta$ and TNF- $\alpha$ did not show any significant differences (data not shown). However, differences in the IL-1 $\beta$ and IL-6 production after $72 \mathrm{~h}$ of infection were observed. The BCG strain MD05c induced higher expression of IL-1 $\beta$ but lower expression of IL- 6 compared to both the noninfected cells and the cells infected with the complemented strain (M::05c) (Fig. 4).

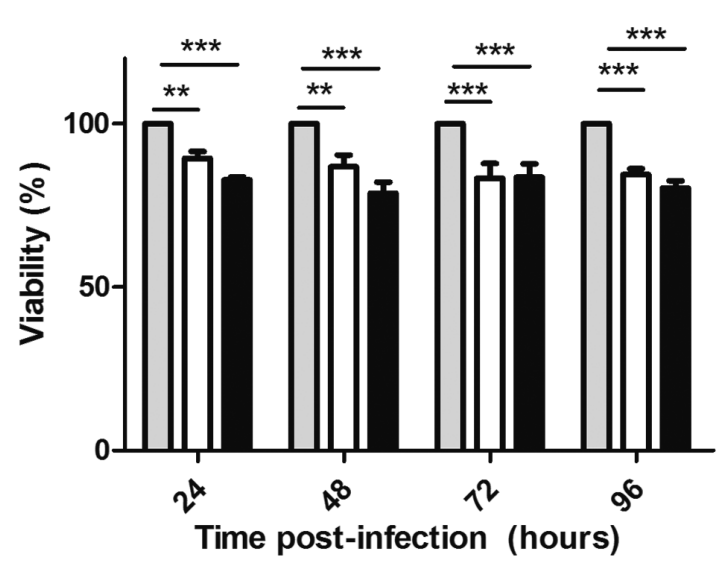

Fig. 3: macrophage viability evaluated with PrestoBlue at 24, 48, 72 and $96 \mathrm{~h}$ post-infection. The data are viability of macrophages (\%) infected with the Bacille Calmette-Guérin (BCG) strains MD05c (white bars) and $\mathrm{M}:: 05 c$ (black bars) relative to non-infected controls (grey bars) and represent the mean $(+/-\mathrm{SD})$ of four independent experiments. Two-way Anova $+/$ SD. Bonferroni post-test **p $<0.01 ; * * * p<0.001$.
In an effort to further characterise the functional impact of mutations mapped in the genome of BCG Moreau, we evaluated two constructs that differ from each other only by the presence of a functional copy of $r v 3405 c$, a transcriptional repressor which regulates $r v 3406$ expression. Rv3406 was characterised as an alkyl sulfatase by Sogi et al. ${ }^{(14)}$ Sulfatases hydrolyse sulfate esters ${ }^{(18)}$ and have been implicated in the modification of the sulfate groups on glycosaminoglycans (GAGs), which are involved in developmental cell signalling and patterning phenomena in the extracellular matrix (ECM) and may play certain roles in bacterial pathogenesis. ${ }^{(15)}$ Additionally, sulfatases are involved in redox reactions and can be considered as alternative sources of sulfur important for biosynthesis of the essential amino acids and sulfurcontaining cofactors. ${ }^{(19)}$

Our results indicate that the absence of the Rv3405c transcriptional repressor confers a growth advantage to the BCG Moreau in the axenic medium and in the intracellular environment of the THP-1 cells compared to the BCG Moreau complemented with a functional copy of $r v 3405 c(\mathrm{M}:: 05 c)$. One explanation is that the absence of $r v 3405 c$ and consequent constitutive expression of Rv3406 [Supplementary data (Fig. 2)] may contribute to enhanced mycobacterial adaptation possibly by conferring a metabolic advantage related to sulfur metabolism and/or carbon acquisition. This protein may be particularly important because it is the only annotated type II sulfatase in $\mathrm{Mtb}^{(14)}$ even though mycobacterial genomes have an unusually high number of genes predicted to encode sulfotransferases and sulfatases. ${ }^{(19)}$ Moreover, recent drug screening studies have identified mutations that inactivate $r v 3405 c$ in the sequenced Mtb strains which are resistant to new classes of compounds, ${ }^{(20,21)}$ implicating Rv3406 in the resistance mechanism. It is important to consider that other genes apart from rv3406 may also be under the regulatory control of Rv3405c, and we are pursuing the studies aiming to map this possible regulatory network under the axenic and intracellular conditions.
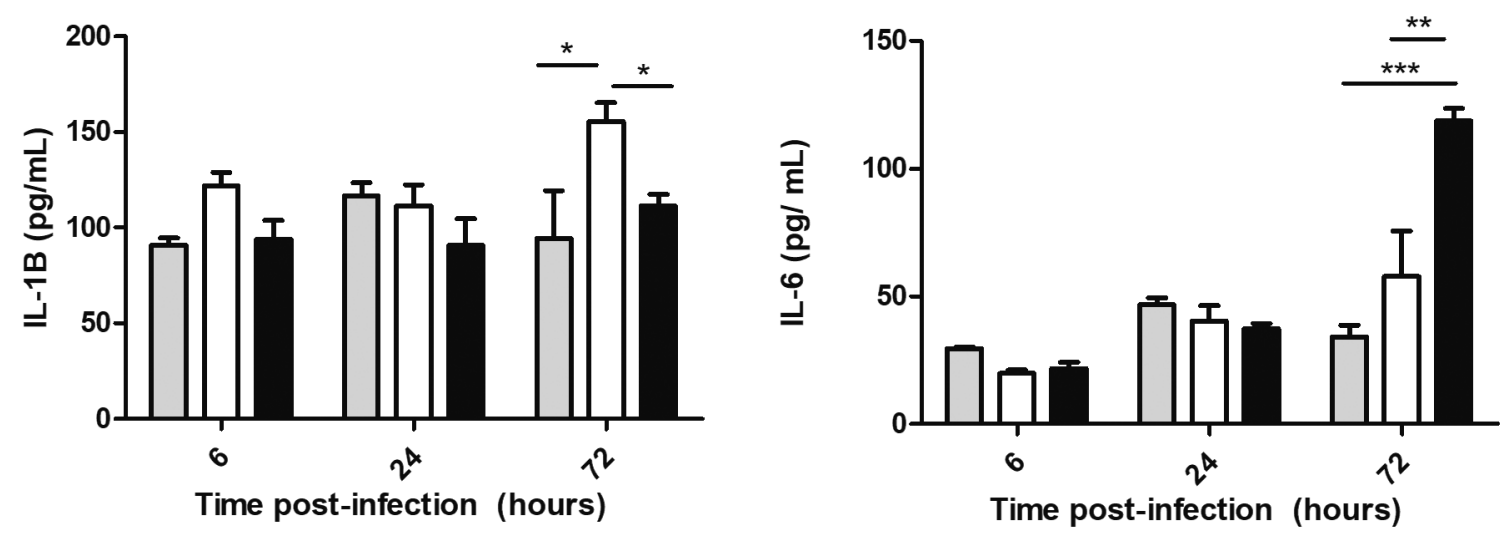

Fig. 4: IL-1 $\beta$ (A) and IL-6 (B) were measured in the culture supernatants from the THP-1 macrophages that were not infected (grey bars) or infected with Bacille Calmette-Guérin (BCG) MD05c (white bars) and M::05c (black bars) at 6, 24 and 72 h post infection by enzyme-linked immunosorbent assay (ELISA). Bars represent the mean (+/- SD) of three independent experiments. Two-way Anova $+/-$ SD. Bonferroni posttest $* \mathrm{p}<0.05 ; * * \mathrm{p}<0.01 ; * * * \mathrm{p}<0.001$. 
Our approach has some limitations, including the use of macrophages derived from a cell lineage, THP-1. Nevertheless, our data provides an insight into the behaviour of BCG in response to the intracellular environment and the behaviour of the host cells in response to the bacterial infection. Protocols for the differentiation of the macrophages from the THP-1 cells are quite diverse in the literature, with variable PMA concentrations of 30 $\mathrm{nM},{ }^{(22)} 80 \mathrm{nM},{ }^{(23)} 100 \mathrm{nM}^{(24)}$ and $200 \mathrm{nM}$; ${ }^{(17)}$ additionally, the protocols differ in the duration of the exposure and post-differentiation incubation time. Lack of a uniform protocol has a strong impact on the interpretation of the results hindering comparison between various studies. Numerous studies have focused on macrophage activation and death in response to $M$. tuberculosis, but much less is known about the BCG-mediated cellular activities in the phagocytic cells. ${ }^{(25)}$ Under the experimental conditions used in our study, significant loss in macrophage viability was observed at all times due to the infection, but no significant loss in macrophage viability was observed due to the recombinant BCG strain used (Fig. 3). Other studies using BCG infected THP-1 cells report similar results. ${ }^{(26,27)}$.

It is well known that cell-mediated immune responses are involved in generating protection against Mtb with broad participation of the $\mathrm{T}_{H} 1$ cells and pro-inflammatory cytokines such as IL-1 $\beta$, IL- 6 , TNF- $\alpha$ and IFN- $\gamma$. These factors increase the microbicidal capacity of the macrophages through production of reactive oxygen and nitrogen intermediates that render the intracellular environment hostile $^{(28)}$ and play a role in the formation of granulomas and subsequent containment of bacilli dissemination. ${ }^{(29)}$

In a previous study, Sousa-Vasconcelos et al. ${ }^{(22)}$ showed that BCG Moreau is capable of increasing the IL-1 $\beta$ and IL-6 levels in the supernatant of the THP-1 cells compared to the non-infected cells. IL-1 $\beta$ is a proinflammatory cytokine involved in the control of bacterial load through the recruitment of microbial agents. ${ }^{(30)}$ It is an important mediator of inflammation and plays a role in generating resistance to the Mtb infections. Our results showed an increase in this cytokine at $72 \mathrm{~h}$ post-infection possibly resulting in a decrease in bacterial viability observed at $96 \mathrm{~h}(\mathrm{p}>0.05)$. IL-6 is a pleiotropic cytokine performing a range of functions related to inflammation, host defence and tissue injury. Additionally, it plays an important role in the metabolism of carbohydrates and lipids by increasing lipolysis and the release of free fatty acids and glycerol. ${ }^{(31)}$ Our data show that IL-6 secretion is higher in the cells infected with the complemented strain $\mathrm{M}:: 05 c$ than in the cells infected with BCG Moreau lacking a functional repressor. Since the presence of the functional repressor abolishes expression of the Rv3406 sulfatase, we could speculate that this loss is partially compensated by the increased expression of IL-6 through its effects on lipid metabolism and release of fatty acids.

Hayashi et al. ${ }^{(32)}$ compared cytokine secretion by the THP-1 cells in response to various BCG strains, reporting higher levels of IL- $1 \beta$ but lower levels of IL- 6 secretion induced by BCG Moreau than by BCG Pasteur. Considering that BCG Pasteur carries a functional Rv3405c repressor, we obtained a similar cytokine pro- file. BCG Moreau and Pasteur differ in numerous genetic loci apart from rv3405c, making it almost impossible to establish the differences that are responsible for each observed phenotype. Our approach involves comparing two BCG Moreau constructs differing only in the presence of an active transcriptional repressor; it provides an opportunity to assess the impact of the loss of Rv3405c on some aspects of BCG Moreau physiology. Overall, our data indicate that constitutive expression of a type II sulfatase, Rv3406, favours bacterial viability and leads to a differential cytokine response in an in vitro model of infection. Increased viability may have a positive impact on vaccine efficacy. This is an example of functional gain due to gene loss that contributes to the detailed characterisation of the $M$. bovis BCG strain used in Brazil for production of vaccine against tuberculosis.

\section{AUTHORS' CONTRIBUTION}

RMM, RTP and LML conceived and designed the experiments; RMM, PRC and PSSV performed the experiments; RMM, PRC, RTP and LML analysed the data. All authors read and contributed to the article.

\section{REFERENCES}

1. WHO - World Health Organization. Global tuberculosis report 2017. Available from: http://www.who.int/tb/publications/global_report/en/,

2. Kaufmann SH. EFIS lecture. Immune response to tuberculosis: how to control the most successful pathogen on earth. Immunol Lett. 2016; 175: 50-7.

3. Mann D, Sant'Anna FM, Schmaltz CAS, Freitas DFS, Rolla VC, Cavalcante SC, et al. Cutaneous tuberculosis and HIV infection at a referral centre in Rio de Janeiro, Brazil. Mem Inst Oswaldo Cruz. 2018; 113(9): e180184.

4. Zhang L, Ru HW, Chen FZ, Jin CY, Sun RF, Fan XY, et al. Variable virulence and efficacy of BCG vaccine strains in mice and correlation with genome polymorphisms. Mol Ther. 2016; 24(2): 398-405.

5. Behr M, Wilson MA, Gill WP, Salamon H, Schoolnik GK, Rane S, et al. Comparative genomics of BCG vaccines by whole genome DNA microarray. Science. 1999; 284(5419): 1520-3.

6. Mostowy S, Tsolaki AG, Small PM, Behr MA. The in vitro evolution of BCG vaccines. Vaccine. 2003; 21(27-30): 4270-4.

7. Calmette A, Guerin C, Negre L, Bocquet A. Sur la vaccination preventive des enfants nouveau-nés contre la tuberculose par le BCG. Ann Inst Pasteur (Paris). 1927; 3: 201-8.

8. Ritz N, Curtis N. Mapping the global use of different BCG vaccine strains. Tuberculosis (Edinb). 2009; 89(4): 248-51.

9. Zhang W, Zhang Y, Zheng H, Pan Y, Liu H, Du P, et al. Genome sequencing and analysis of BCG vaccine strains. PLoS One. 2013; 8(8): e71243.

10. Abdallah AM, Hill-Cawthorne GA, Otto TD, Coll F, Guerra-Assunção JA, Gao G, et al. Genomic expression catalogue of a global collection of BCG vaccine strains show evidence for highly diverged metabolic and cell-wall adaptations. Sci Rep. 2015; 5: 15443.

11. Benévolo-de-Andrade TC, Monteiro-Maia R, Cosgrove C, Castello-Branco LRR. BCG Moreau Rio de Janeiro - An oral vaccine against tuberculosis - Review. Mem Inst Oswaldo Cruz. 2005; 100(5): 459-65.

12. Gomes LH, Otto TD, Vasconcellos EA, Ferrão PM, Maia RM, Moreira AS, et al. Genome sequence of Mycobacterium bovis BCG Moreau, the Brazilian vaccine strain against tuberculosis. J Bacteriol. 2011; 193(19): 5600-1. 
13. Galvão TC, Lima CR, Gomes LH, Pagani TD, Ferreira MA, Gonçalves AS, et al. The BCG Moreau RD16 deletion inactivates a repressor reshaping transcription of an adjacent gene. Tuberculosis (Edinb). 2014; 94(1): 26-33.

14. Sogi KM, Gartner ZJ, Breidenbach MA, Appel MJ, Schelle MW, Bertozzi CR. Mycobacterium tuberculosis Rv3406 is a type II alkyl sulfatase capable of sulfate scavenging. PLoS One. 2013; 8(6): e65080.

15. Hanson SR, Best MD, Wong CH. Sulfatases: structure, mechanism, biological activity, inhibition, and synthetic utility. Angew Chem Int Ed Engl. 2004; 43(43): 5736-63.

16. da Cruz FW, McBride AJ, Conceição FR, Dale JW, McFadden J, Dellagostin OA. Expression of the B-cell and T-cell epitopes of the rabies virus nucleoprotein in Mycobacterium bovis BCG and induction of an humoral response in mice. Vaccine. 2001; 20(5-6): 731-6.

17. Daigneault M, Preston JA, Marriott HM, Whyte MK, Dockrell $\mathrm{DH}$. The identification of markers of macrophage differentiation in PMA-stimulated THP-1 cells and monocyte-derived macrophages. PLoS One. 2010; 5(1): e8668.

18. Hossain MM, Kawarabayasi Y, Kimura M, Kakuta Y. Expression and functional analysis of a predicted AtsG arylsulphatase identified from Mycobacterium tuberculosis genomic data. J Biochem. 2009; 146(6): 767-9.

19. Mougous JD, Green RE, Williams SJ, Brenner SE, Bertozzi CR. Sulfotransferases and sulfatases in Mycobacteria. Chem Biol. 2002; 9(7): 767-76.

20. Neres J, Hartkoorn RC, Chiarelli LR, Gadupudi R, Pasca MR, Mori G, et al. 2-Carboxyquinoxalines kill Mycobacterium tuberculosis through noncovalent inhibition of DprE1. ACS Chem Biol. 2015; 10(3): 705-14.

21. Tiwari D, Park SW, Essawy MM, Dawadi S, Mason A, Nandakumar M, et al. Targeting protein biotinylation enhances tuberculosis chemotherapy. Sci Transl Med. 2018; 10(438): pii: eaal1803.

22. Sousa-Vasconcelos PS, Seguins WS, Luz ES, de Pinho RT. Pattern of cytokine and chemokine production by THP-1 derived macro- phages in response to live or heat-killed Mycobacterium bovis bacillus Calmette-Guérin Moreau strain. Mem Inst Oswaldo Cruz. 2015; 110(6): 809-13.

23. Guerreiro LT, Robottom-Ferreira AB, Ribeiro-Alves MG, Brito TR, Rosa PS, Sandoval FG, et al. Gene expression profiling specifies chemokine, mitochondrial and lipid metabolism signatures in leprosy. PLoS One. 2013; 8(6): e64748.

24. Mendoza-Coronel E, Castañón-Arreola M. Comparative evaluation of in vitro human macrophage models for mycobacterial infection study. Pathog Dis. 2016; 74(6): pii: ftw052.

25. Chávez-Galán L, Vesin D, Martinvalet D, Garcia I. Low dose BCG infection as a model for macrophage activation maintaining cell viability. J Immunol Res. 2016; 17 pp.

26. Riendeau CJ, KornfeldH. THP-1 Cell apoptosis in response to mycobacterial infection. Infect Immun. 2003; 71(1): 254-9.

27. Hasan Z, Ashraf M, Tayyebi A, Hussain R. M. leprae inhibits apoptosis in THP-1 cells by downregulation of Bad and Bak and upregulation of Mcl-1 gene expression. BMC Microbiology. 2006; 6: 78.

28. Cooper AM, Khader SA. The role of cytokines in the initiation, expansion, and control of cellular immunity to tuberculosis. Immunol Rev. 2008; 226: 191-204.

29. Lin PL, Flynn JL. Understanding latent tuberculosis: a moving target. J Immunol. 2010; 185(1): 15-22.

30. Jayaraman P, Sada-Ovalle I, Nishimura T, Anderson AC, Kuchroo VK, Remold HG, et al. IL- $1 \beta$ promotes antimicrobial immunity in macrophages by regulating TNFR signaling and caspase- 3 activation. J Immunol. 2013; 190(8): 4196-204.

31. Volp ACP, Alfenas RCG, Costa NMB, Minim VPR, Stringueta PC, Bressan J. Capacidade dos biomarcadores inflamatórios em predizer a síndrome metabólica: inflammation biomarkers capacity in predicting the metabolic syndrome. Arq Bras Endocrinol Metabo. 2008; 52(3): 537-49

32. Hayashi D, Takii T, Fujiwara N, Fujita Y, Yano I, Yamamoto S, et al. Comparable studies of immunostimulating activities in vitro among Mycobacterium bovis bacillus Calmette-Guérin (BCG) substrains. FEMS Immunol Med Microbiol. 2009; 56(2): 116-28. 